\title{
Erosive lichen planus: a therapeutic challenge ${ }^{*}$
}

\author{
Williams Romero ${ }^{1}$ \\ Lucas Navajas-Galimany ${ }^{1}$
}

\author{
Laura Giesen $^{1}$ \\ Sergio Gonzalez ${ }^{1}$
}

DOI: http:/ / dx.doi.org/10.1590/abd1806-4841.20164046

\begin{abstract}
Erosive lichen planus is an uncommon variant of lichen planus. Chronic erosions of the soles, accompanied by intense and disabling pain, are some of its most characteristic manifestations. We present the case of a woman who developed oral and plantar erosive lichen planus associated with lichen planus pigmentosus and ungueal lichen planus that were diagnosed after several years. The patient failed to respond to multiple therapies requiring longstanding medication but remained refractory. Knowledge of the treatment options for erosive lichen planus is insufficient. Further research is required to clarify their effectiveness, ideally adopting an evidence-based methodology.
\end{abstract}

Keywords: Cyclosporine; Lichen planus; Methotrexate; Steroids; Tacrolimus

\section{INTRODUCTION}

Erosive lichen planus (ELP) is a very uncommon variant of lichen planus with mucosal, palm and/or plantar involvement. It usually presents with chronic erosion of the soles, accompanied by intense, disabling pain and progressive loss of toenails. Multiple treatment options have been proposed, but none of them has consistently demonstrated remission of lesions. We present the case of a woman with longstanding erosive plantar lesions, highlighting the difficulty in making an early diagnosis and achieving clinical response.

\section{CASE REPORT}

A healthy, 75 year-old woman visited our outpatient clinic after 10 years of progressive destruction of her toenails and emergence of painful lesions on both heels, which later became erosive. Oral lesions appeared 5 years later and during the preceding 6 months she had noted progressive appearance of pruritic lesions on the groins and back. On physical ex- amination, the patient presented with well-defined extensive erosions on both heels; erosive plaques on the buccal mucosa; hyperpigmented lichenoid papules on the groins and back; destruction of the ungueal lamina and dorsal pterygium on her feet (Figures 1 and 2). Laboratory testing showed no findings and serologic studies for HIV, HCV and HBV were negative. The histopathological study revealed orthoqueratosis, epidermic atrophy, vacuolar degeneration of basal keratinocytes, neutrophil exocytosis, Civatte bodies and dense perivascular superficial infiltrate (Figure 3 ). These findings, together with the clinical manifestations, confirmed the diagnosis of oral and plantar erosive lichen planus associated with lichen planus pigmentosus and ungueal lichen planus. We started treatment with systemic corticosteroids (prednisone $1 \mathrm{mg} / \mathrm{kg}$ ), topical treatment on both heels using clobetasol $0.05 \%$ interchanged with tacrolimus $0.1 \%$ twice daily, as well as oral topical treatment with betamethasone and tetracycline. Due to a complete lack

\footnotetext{
Received on 16.09 .2014

Approved by the Advisory Board and accepted for publication on 04.10.2014

Work performed at the Pontifical Catholic University of Chile - Santiago, Chile.

Financial Support: None.

Conflict of Interest: None.

1 Pontificia Universidad Católica de Chile - Santiago, Chile.

(C)2016 by Anais Brasileiros de Dermatologia
} 


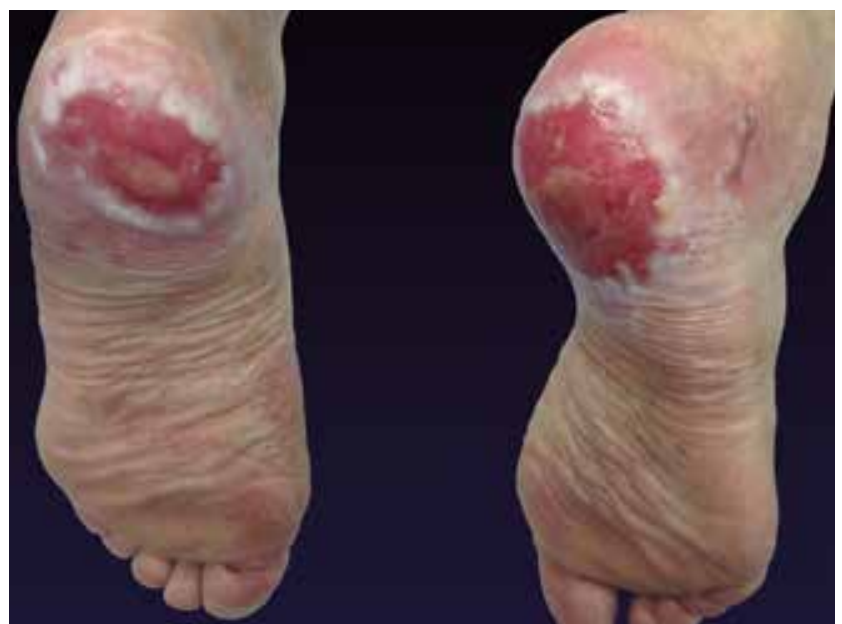

Figure 1: Brilliant erythematous plaques with extensive superficial erosions on both heels

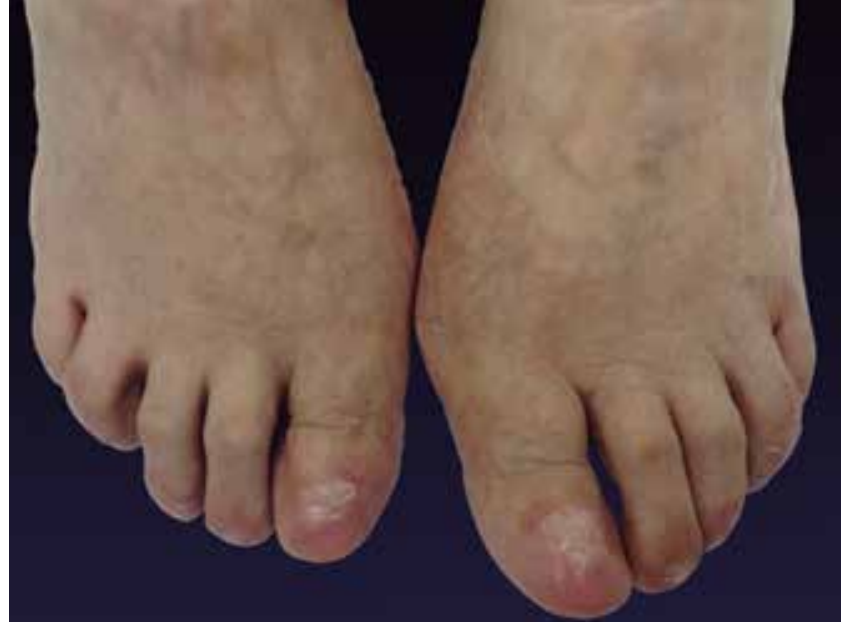

FIGURE 2: Destruction of ungueal lamina, with anonychia and dorsal pterygium on both feet

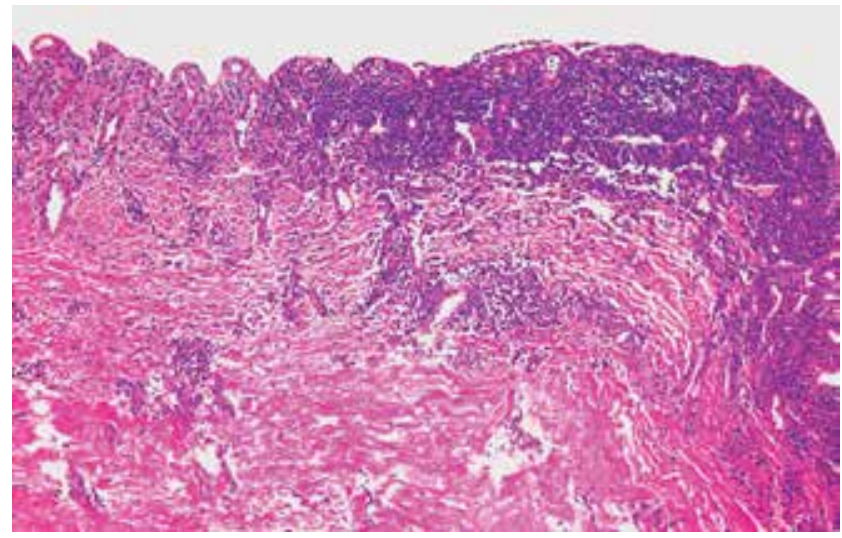

Figure 3: Microphotograph showing denuded skin with lymphocytic lichenoid superficial infiltrates and fibrosis. HE, 100x

of response, cyclosporine was initiated at a dosage of $50 \mathrm{mg}$ three times a day. The patient's blood pressure increased without improvement in skin lesions, so cyclosporine was discontinued and methotrexate was started at a dosage of $15 \mathrm{mg} /$ week. Thus far, the patient has undergone follow-up for approximately five months, taking up to $20 \mathrm{mg} /$ week of methotrexate and only partial regression has been observed. We recently discontinued methotrexate and treatment using thalidomide was proposed but the patient decided to stop all treatments and keep only plastic dressings with moisturizers on her feet to walk and avoid more damage.

\section{DISCUSSION}

ELP is a very uncommon variant of lichen planus. ${ }^{1}$ It is characterized by painful erosions of torpid evolution, mainly localized in the plants, palms, scalp, oral and genital mucosa, as well as typical lichen planus lesions in the nails, mucous membranes and skin. ${ }^{2}$ The natural course of this disorder usually begins with erythematous plaques and blisters on the feet, which develop into painful and disabling erosions, predominantly on the heels. Progressive toenail destruction and dorsal pterygium, as well as painful erosions on the scalp, oral and genital mucosa, are characteristic as the disease develops. ${ }^{3}$ It is frequently accompanied by a significant functional impairment. Malignant transformation to squamous cell carcinoma has been described as one of its main and latest complications.

The etiology of lichen planus remains uncertain. The role of immune cellular response, especially of T-cell lymphocytes and macrophages, has proven to be the mainstay of its pathogenesis. ${ }^{4}$ T-cells may be responsible for keratinocyte apoptosis and intensifying inflammatory response. ${ }^{5}$ Among biopsies from patients with ELP, TNF- $\alpha$ was over-expressed in the cytoplasm of the basal epithelial keratinocytes. ${ }^{6,7}$

Owing to its chronic and refractory course, multiple treatment options have been proposed, with variable results: systemic, local and intralesional glucocorticoids, retinoids, cyclosporine, azathioprine, phototherapy, dapsone, thalidomide, tacrolimus, among others. Although treatment is still controversial, topical corticosteroids and topical calcineurin inhibitors are considered treatments of choice for localized ELP forms. Both were tested in this case and none led to clinical improvement. A systematic review published in 2012 intended to assess the evidence supporting systemic therapies for ELP. ${ }^{8}$ A total of 420 ELP cases were identified. Systemic corticosteroid treatment was the most commonly used ( $27 \%$ of all cases) and the second most effective in reducing lesion count and symptoms. Among systemic retinoids, acitretin exhibited the poorest performance, whereas both etretinate and isotretinoin entailed significant pain relief and healing of erosions. Other common treatments 
were immunosuppressant drugs, which were used in $8.87 \%$ of cases. Azathioprine appeared to be the most effective, with complete resolution observed in $75 \%$ of cases and no recurrence reported up to 9 months after discontinuation. Thalidomide yielded complete resolution of lesions in $50 \%$ of cases. Cyclosporine produced near-complete resolution during therapy but presented a high recurrence rate once treatment was discontinued. Importantly, this report lacks randomized controlled trials; it is based mainly on expert opinions and case reports with very low-quality evidence. Moreover, only a few studies describe treatment options for the plantar variant, making results even more difficult to analyze. Extracorporeal phototherapy has proven to be effective in T-cell mediated diseases and has been successfully tested in recalcitrant forms of ELP. ${ }^{9}$ Low molecular weight heparin at low doses also demonstrated good response, acting as an inhibitor of the hypersensitivity reaction involved. ${ }^{4}$ Another treatment alternative is surgery, alone or together with cyclosporine, which has been effective in patients with chronic, stable and localized lesions..$^{10}$ In this case, our patient did not respond to corticosteroids, cyclosporine nor methotrexate; she decided to stop all treatments and keep only plastic dressings with moisturizers on her feet.

In conclusion, this is an uncommon, chronic disorder, with a refractory course. We stress the difficulty of establishing an early diagnosis and thus the importance of high suspicion aroused by any erosive lesion of torpid evolution and characteristic localization. Treatment is still controversial, while topical corticosteroids and topical calcineurin inhibitors are the treatments of choice for localized forms. Short pulses of systemic glucocorticoids, phototherapy, and systemic immunosuppressors are available for generalized cases. Further research is required to clarify their effectiveness, ideally drawing on an evidence-based methodology. Meanwhile, prescriptions should be individualized according to comorbidities, previous treatments, drug side effects and clinical experience. Long-term monitoring of all patients is also very important, allowing early detection of a malignant tumor.]

\section{REFERENCES}

1. Rebora A. Erosive Lichen planus: what is this? Dermatology. 2002;205:226-8.

2. Cram DL, Kierland RR, Winkelmann RK. Ulcerative lichen planus of the feet. Arch Dermatol. 1966;93:692-701.

3. García Peŕez A, Rodríguez Pichardo A, Bueno Montes J. Liquen plano erosivo plantar con oni-coatrofia. Med Cutan Ibero Lat Am. 1982;10:89-92.

4. Jiménez-Sánchez MD, Ferrándiz L, Moreno-Ramírez D, Vallejo-Benítez A Camacho-Martínez F. Liquen plano erosivo plantar. Actas Dermosifiliogr. 2012;103:448-50.

5. Sklavounou-Andrikopoulou A, Chrysomali E, lakovou M, Garinis GA, Karameris A Elevated serum levels of the apoptosis related molecules TNF-alpha, Fas/Apoand Bcl-2 in oral lichen planus. J Oral Pathol Med. 2004;33:386-90.

6. Zhou G, Xia K, Du GF, Chen XM, Xu XY, Lu R, et al. Activation of nuclear factorkappa $B$ correlates with tumor necrosis factor-alpha in oral lichen planus: a clinicopathologic study in atrophic-erosive and reticular form. J Oral Pathol Med. 2009;38:559-64

7. Porter SR, Kirby A, Olsen I, Barrett W. Immunologic aspects of dermal and oral lichen planus: a review. Oral Surg Oral Med Oral Pathol Oral Radiol Endod. 1997;83:358-66

8. Kou Ho J, Hantash B. Systematic review of current systemic treatment options for erosive lichen planus. Expert Rev Dermatol. 2012;7:269-82.

9. Elewa R, Altenburg A, Zouboulis CC. Recalcitrant severe erosive cutaneous lichen planus treat-ed with extracorporeal photopheresis monotherapy. $\mathrm{Br} \mathrm{J}$ Dermatol. 2011;165:441-3.

10. Patrone P, Stinco G, La Pia E, Frattasio A, De Francesco V. Surgery and cyclosporine $A$ in the treatment of erosive lichen planus of the feet. Eur J Dermatol. 1998:8:243-4.

\author{
MAILING ADDRESS: \\ Lucas Navajas Galimany \\ Av. Vicuña Mackenna 4686. \\ Santiago, Chile \\ E-mail:Ifnavaja@uc.cl
}

How to cite this article: Romero W, Giesen L, Navajas-Galimany L, Gonzalez S. Erosive lichen planus: a therapeutic challenge An Bras Dermatol. 2016;91(1):84-6. 